Supporting Information

\title{
One Single Graphene Oxide Film for Responsive
}

\section{Actuation}

Huhu Cheng, ${ }^{\dagger,+}$ Fei Zhao, ${ }^{\dagger}$ Jiangli Xue, ${ }^{\dagger}$ Gaoquan Shi, ${ }^{*}$ Lan Jiang ${ }^{\S}$ and Liangti Qu ${ }^{\dot{\dagger} *}$

${ }^{\dagger}$ Beijing Key Laboratory of Photoelectronic/Electrophotonic Conversion Materials, Key

Laboratory of Cluster Science, Ministry of Education of China, School of Chemistry, Beijing Institute of Technology, Beijing 100081, P. R. China.

tDepartment of Chemistry, Tsinghua University, Beijing 100084, P. R. China

${ }^{\S}$ Laser Micro-/Nano-Fabrication Laboratory, School of Mechanical Engineering, Beijing Institute of Technology, Beijing 100081, P. R. China

*Email:1qu@bit.edu.cn.

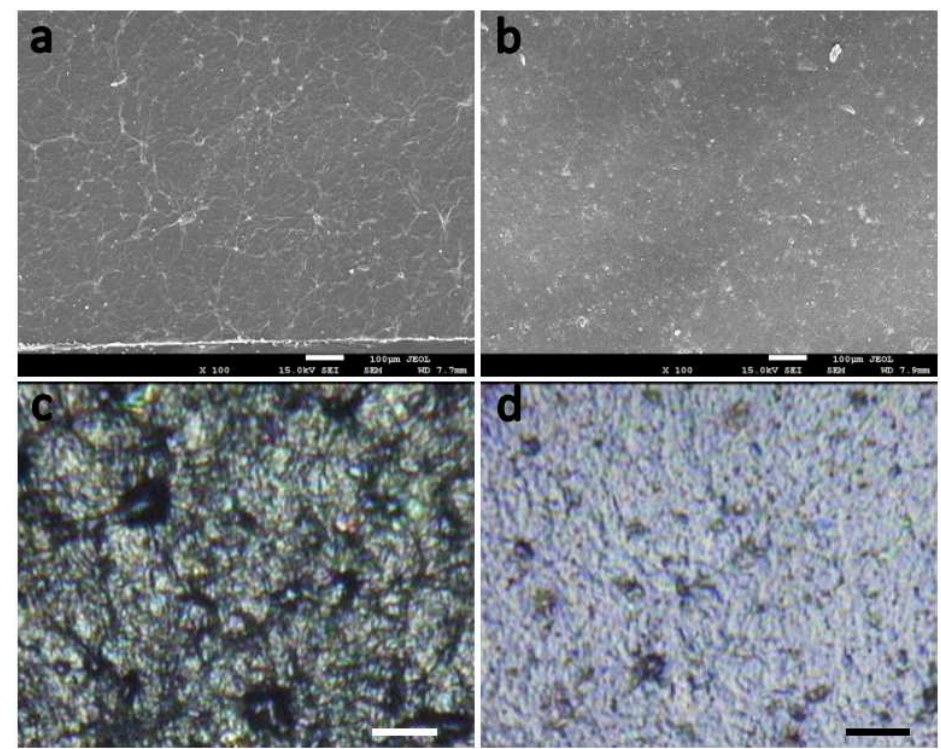


Figure S1. (a) and (b) are the SEM images of rough surface and smooth surface of GO film after magnifying 100 times, respectively. (c) and (d) are the optical microscope images of rough surface and smooth surface of GO film, respectively. Scale bars: a and b, $100 \mu \mathrm{m}$; $\mathrm{c}$ and $\mathrm{d}, 500 \mu \mathrm{m}$.
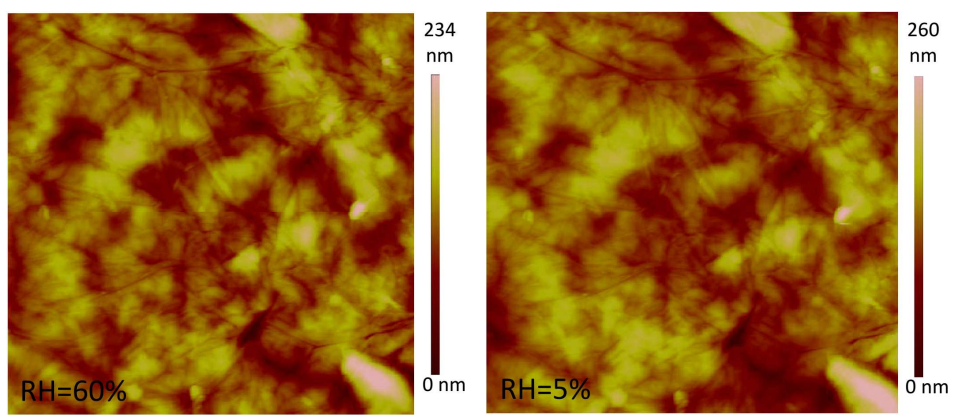

Figure S2. In-situ AFM images $(20 \times 20 \mu \mathrm{m})$ of the smooth surface of the GO film under different relative humidity.

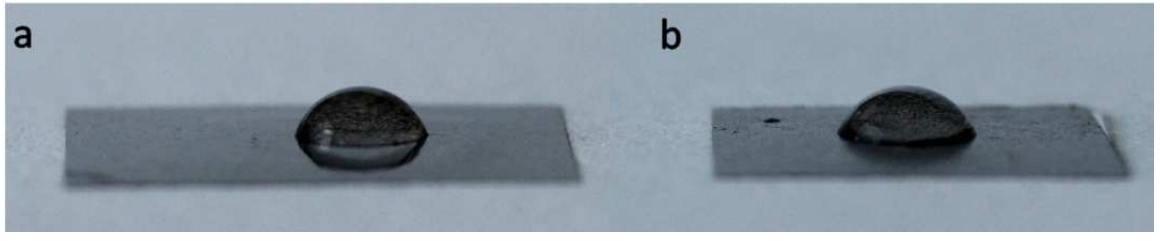

Figure S3. Photographs of a drop of water on the shiny surface (a) and rough surface (b) of the GO film.

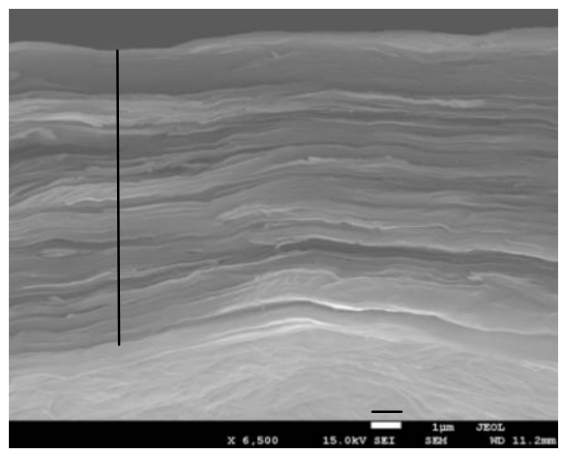

Figure S4. SEM image of the cross section of the GO film. 

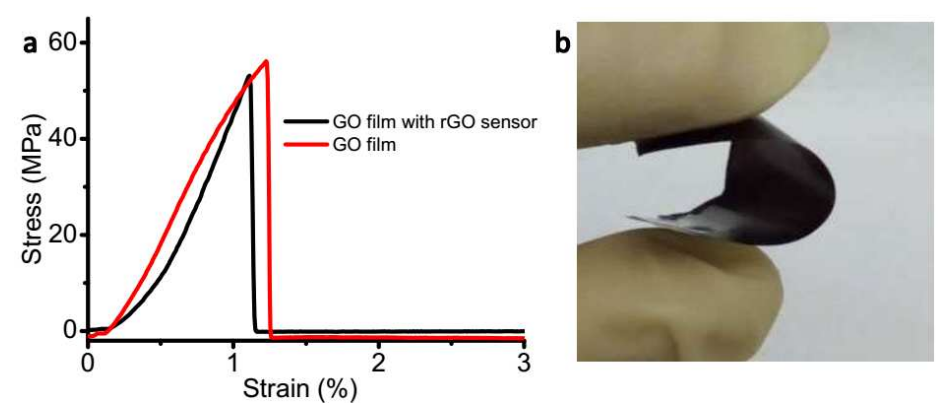

Figure S5. (a) The stress-strain curve of GO film and GO film with built-in rGO sensor. (b) Photo of the film under bending state.
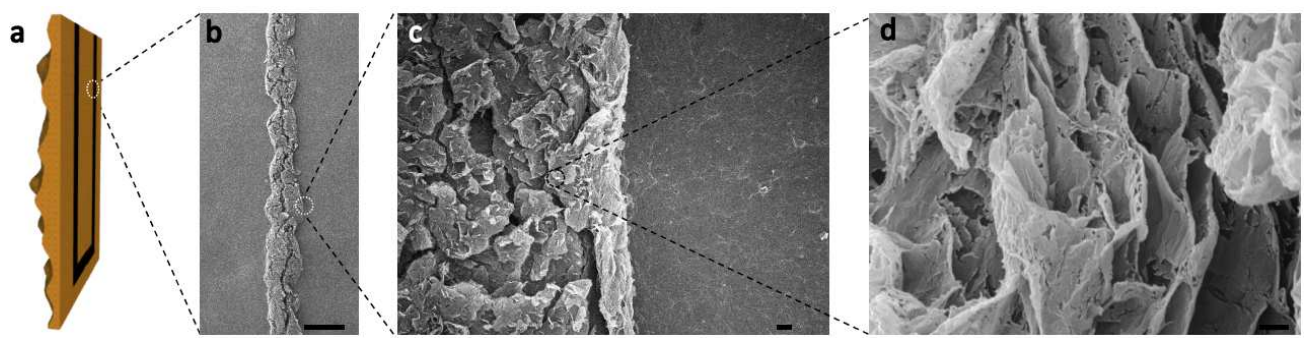

Figure S6. (a) Schematic of the film with laser writing rGO circuit as built-in sensor. (b-d) SEM images of the laser writing rGO circuit in the film with different multiples. Scale bars: $b$, $0.5 \mathrm{~mm} ; \mathrm{c}, 10 \mu \mathrm{m} ; \mathrm{d}, 1 \mu \mathrm{m}$.

Figure S6 shows the structure in the laser writing rGO circuit (Figure S6b). The width of rGO circuit is about $0.4 \mathrm{~mm}$. rGO sheets in the circuit has porous structure (left region in Figure S6c and 6d) and the GO is still flat (right region in Figure S6c).

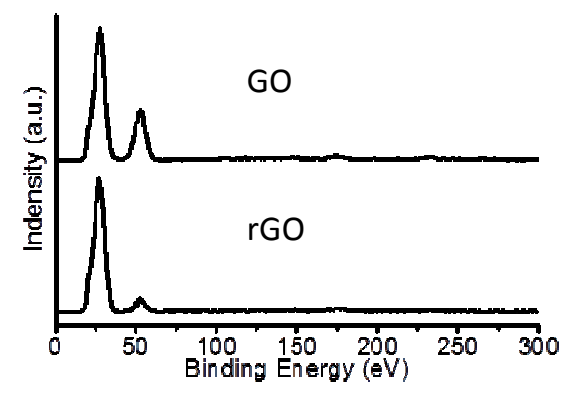

Figure S7. EDS of the GO and laser writing rGO circuit in the film, respectively. 


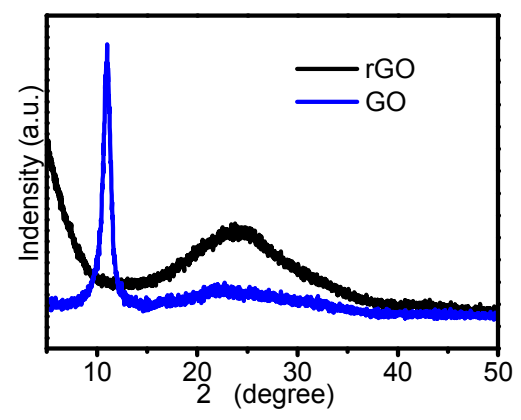

Figure S8. XRD of the GO and laser writing rGO, respectively.
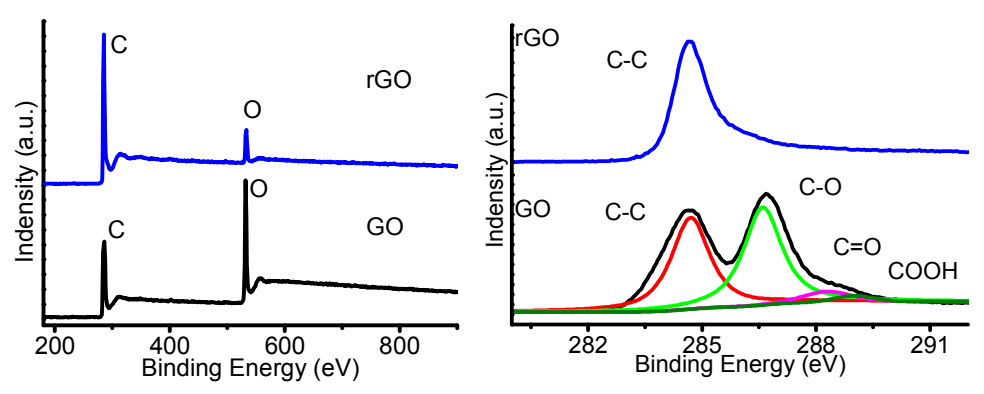

Figure S9. (a) XPS of the GO and laser writing rGO. (b) The high-resolution $\mathrm{C} 1 \mathrm{~s}$ spectrum of the GO and rGO.

X-ray diffraction (XRD) analysis on a laser writing rGO thin film under the same conditions to those applied for laser writing rGO circuit, verifying that the peak of GO $(2 \theta=$ $11^{\circ}$, Figure S8) has completely disappeared, while the rGO has a representative graphene peak at about $24^{\circ}$. X-ray photoelectron spectroscopy (XPS) results further confirm that the intensity of O peak for rGO has decreased drastically in comparison with GO (Figure S9), which agrees with the EDS result mentioned above. The high-resolution $\mathrm{C}$ 1s spectrum of the rGO reveals the $\mathrm{C}-\mathrm{C}$ bonds $(\sim 284.8 \mathrm{eV})^{\mathrm{s} 1, \mathrm{~s} 2}$ become dominant compared with the GO, indicating the efficient reduction of GO by this laser directly writing process. 


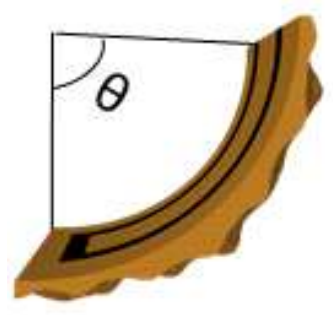

Figure S10. Schematic of the degree of the film bending curvature. The curvature change $(\theta)$ is used to evaluate the actuation performance.
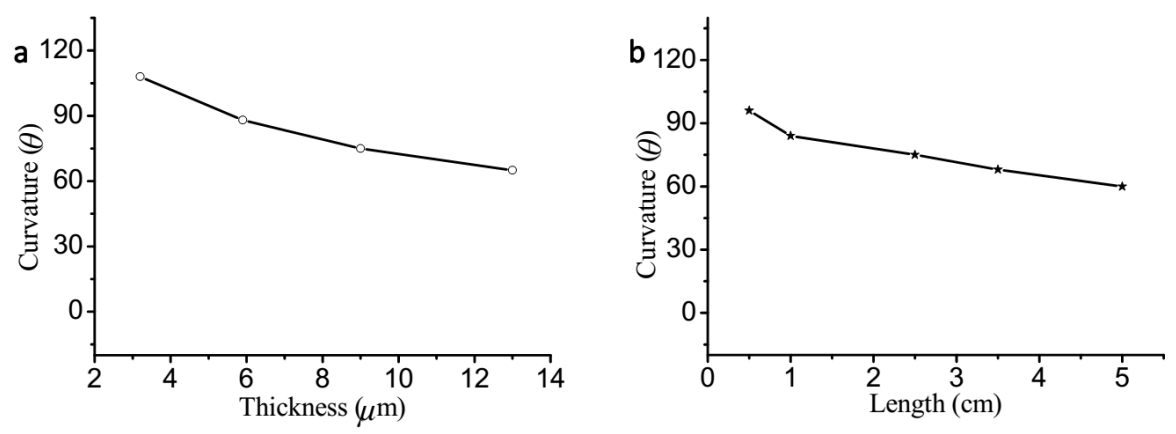

Figure S11. (a) The curve of curvature and thickness of the film. (b) The curve of curvature and length of the film. 

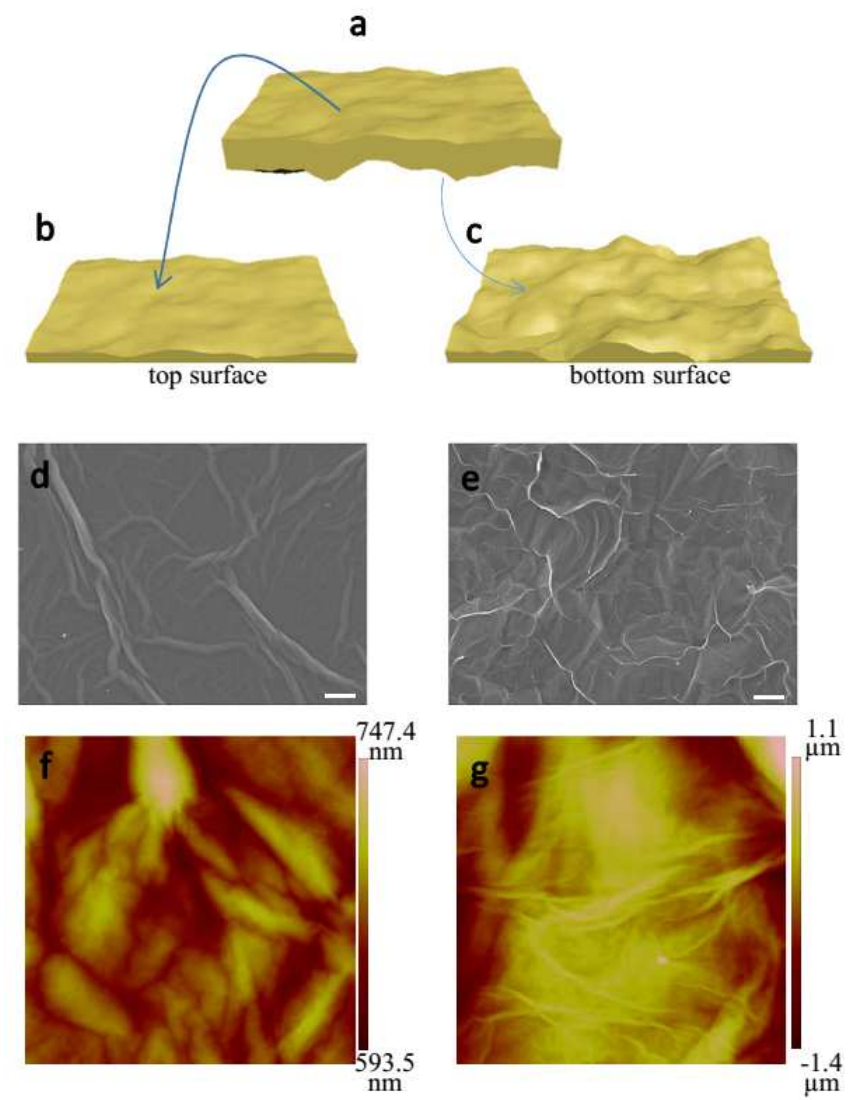

Figure S12. (a-c) Schematic of GO film with relative rough surface (c) on the bottom side when coating the GO solution on a commercial sandpaper (6000 mesh). (d) SEM image of top surface and bottom surface (c). (f) AFM image $(50 \times 50 \mu \mathrm{m})$ of the top surface and (g) bottom surface.

The curvature $(\theta)$ becomes a little smaller when the thickness or the length of the sample increases (Figure S11), indicating the thickness and shapes would affect the mass of the film that further affect the actuation performance. The asymmetric responsiveness of the asymmetric GO film mainly depends on the roughness difference on the surface. When coating the GO suspension on a commercial sandpaper (6000 mesh), the obtained film (Figure S12) will have a coarser surface at the bottom surface (Figure S12c, e and g), and the roughness is about $322 \mathrm{~nm}$, while the top surface roughness is about $194 \mathrm{~nm}$ (Figure S12b, d and $\mathrm{f}$ ). When the $\mathrm{RH}$ is at $85 \%$, the film will bend to the top surface which is relatively smooth. In other words, the film will bend to the relatively smooth side under high RH no 
matter whether the relatively smooth surface of GO film is at the top or bottom side in the preparation process. The results further reveal the roughness difference is the main reason for the asymmetric responsiveness of the asymmetric GO film.

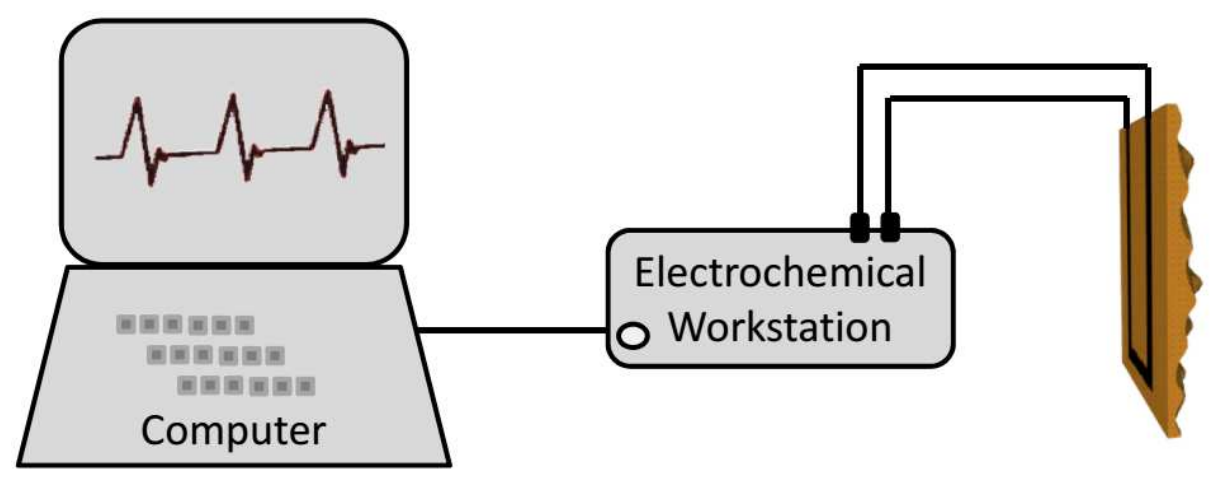

Figure S13. Schematic of detecting electrical signal changes of the built-in rGO sensor in real time of GO film's deformation.

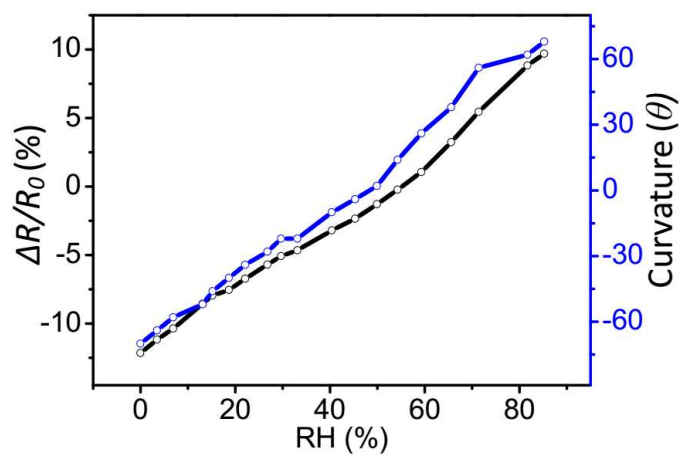

Figure S14. The curvature of the film $(\theta$, blue line) and electrical resistance changes of the built-in $\mathrm{rGO}$ sensor $\left(\Delta R / R_{0}\right.$, black line $)$ along with the $\mathrm{RH}$ changes. 

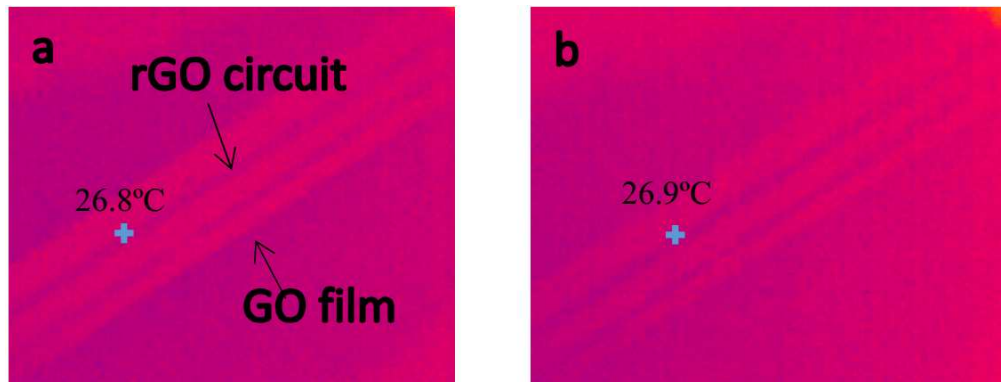

Figure S15. The thermal images of a GO film with rGO circuit sensor before (a) and after (b) the applied voltage $(0.1 \mathrm{~V})$ on it.

In the testing process, the applied voltage would generate heat that affect the actuation of the film. Futher research indicate that the temperature has no change before and after the applied voltage $(0.1 \mathrm{~V})$ on the sample. The reason could be that the value of current flow $(\sim 6.2 \mu \mathrm{A})$ is so small that it cannot generate enough heat to change the temperature on the device.

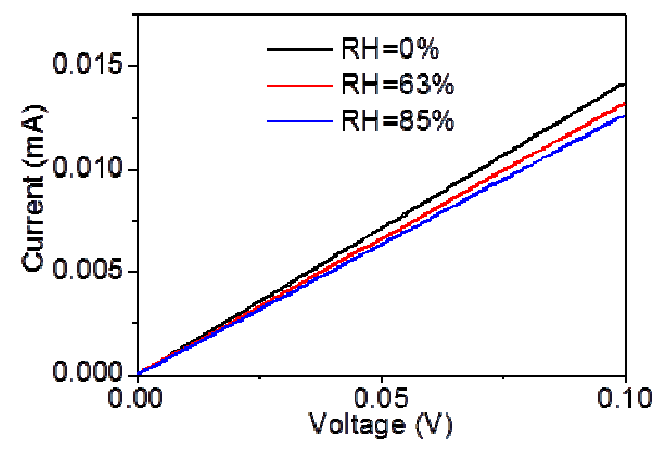

Figure S16. The voltage-current curves of built-in rGO sensor in a fixed film when exposed to different relative humidity. The applied voltage is $0.1 \mathrm{~V}$. The slop of the curve decrease along with the humidity increase means that the electrical resistance of the rGO sensor increases. 

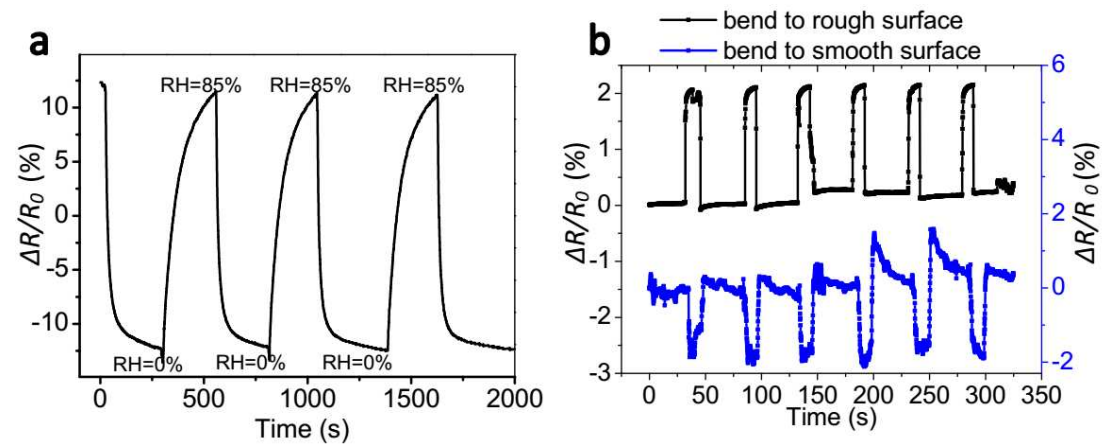

Figure S17. (a) The electrical resistance changes of the built-in sensor induced under the RH alteration when fixed the GO film. (b) The electrical resistance changes of the built-in sensor when bending the film to the smooth (blue line) or rough (black line) surface without humidity change.
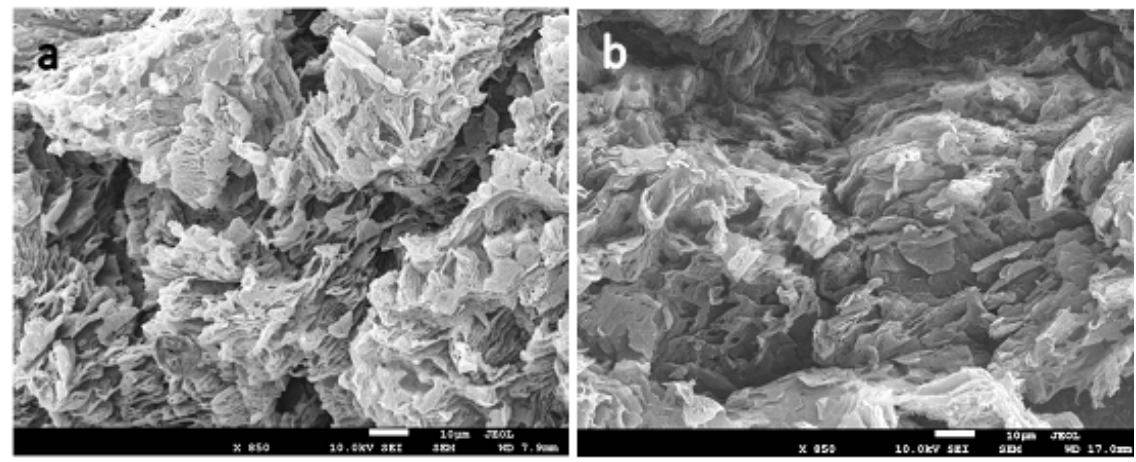

Figure S18. SEM images of the rGO circuit when bending the film to the rough side (a) and smooth side (b). The sheets in rGO circuit compact together when bending to the smooth side. 


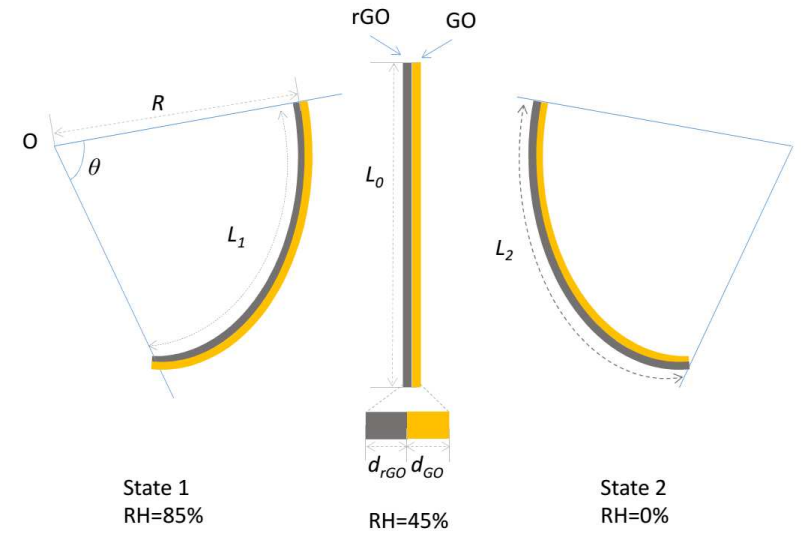

Figure S19. Schematic representation of the bending angel of the film with correlative parameters for calculating the expected strain.

Under high $\mathrm{RH}$, the freestanding film will bend to the smooth side. The electrical resistance of the rGO sensor increases along with the RH increase (Figure S16). The increased degree of electrical resistance of the built-in sensor in a fixed film after RH increase is about $13.8 \%$ (Figure S17a). When the humidity is constant, the bending of GO film to the smooth side will make the sheets in rGO circuit compact together (Figure S18), leading to the electrical resistance decrease of about $-2.1 \%$ (blue line in Figure S17b). So the electrical resistance change of the built-in rGO sensor in Figure $3 \mathrm{e}$ in the main text is the result of a combination of the film deformation and surrounding humidity changes.

The expected strain on the surface of rGO film under bending state could be another factor that is attribute to the electrical resistance changes. The laser reduction process builds rGO layer of about $5 \mu \mathrm{m}$ depth on the GO film in the circuit region. Figure S19 shows the schematic of the cross section of the sample composed of rGO and GO layers. At high RH $(85 \%)$ in Figure $3 \mathrm{~d}$, the rGO is under compressive strain (state 1 in Figure $\mathrm{S} 9$ ). At low $\mathrm{RH}$, the rGO is under tensile strain (state 2). To quantitatively express the expected strain of the rGO, we introduce several essential parameters as follows in Figure S19: ${ }^{\mathrm{3}}$

$\theta$ : Angle (degree) of curvature of the film under bending state. 
$L_{x}$ : the length of the film. $L_{0}$ stands for the originallength of film. $L_{l}$ stands for the length of the rGO layer under compressive strain at state $1 . L_{2}$ stands for the length of the rGO layer under tensile strain at state 2 .

$\Delta L$ : Length change of the rGO layer.

$d x$ : Thickness of the constitutive layers. $d_{r G O}$ and $d_{G O}$, standing for rGO or GO layer.

$R$ : The radius of curvature of the bending motion, which is measured from the base point $\mathrm{O}$ to the midcourt line of inner side layer.

Tentatively ignoring the interaction between the two layers, we have the equations (1), (2), and (3) based on the relationship between the angle of curvature $(\theta)$ and arc length.

$$
\begin{aligned}
& \mathrm{L}_{0}=R \frac{\pi \theta}{180^{\circ}} \\
& \mathrm{L}_{1}=\left(R-d_{r G O}\right) \frac{\pi \theta}{180^{\circ}} \\
& \mathrm{L}_{2}=(2) \\
& \left(R+d_{G O}+d_{r G O}\right) \frac{\pi \theta}{180^{\circ}}
\end{aligned}
$$

Accordingly, the expected strain of the rGO under compressive strain at state 1 can be deduced to be:

$\left(\mathrm{L}_{1}-\mathrm{L}_{0}\right) / \mathrm{L}_{0}=-d_{r G O} \frac{\pi \theta}{180^{\circ}} / L_{0} \times 100 \%$

The expected strain of the rGOunder tensile strain at state 2 can be deduced to be:

$\left(L_{2}-L_{0}\right)=\left(d_{G O}+d_{r G O}\right) \frac{\pi \theta}{180^{\circ}} / L_{0} \times 100 \%$

Therefore, in principle, the expected strain of the rGO could be roughly estimated by measuring the thickness of the rGO layer $\left(d_{r G O}\right)$, GO layer $\left(d_{G O}\right)$ and the angle (degree) of curvature of the film $(\theta)$ under bending state.The thickness for rGO layer within the film is $c a .5 \mu \mathrm{m}$. The $\theta$ under high $\mathrm{RH}(85 \%)$ is about $75^{\circ}$ at state 1 in Figure 3.The $\theta$ under low $\mathrm{RH}$ $(0 \%)$ is about $70^{\circ}$ at state 2 . According to the deduced formula (4) and (5), we thus can roughly figure out the expected compressive strain and thetensile strain of the rGO is about - 
$0.26 \%$ and $0.44 \%$, respectively. This could not cause the intrinsic resistance change of rGO that has been demonstrated in the literature. ${ }^{\mathrm{s}, 5}$

Thus, the sensing property of rGO could mainly attribute to the inter-sheet resistance change. Due to the presence of small amounts of oxygen containing functional groups on the rGO sheets (Figure S7), the water molecules could cause an increase in the distancebe tween the rGO layers at high $\mathrm{RH}^{\mathrm{s} 6,7}$ resulting an increased degree of electrical resistance. Otherwise, the bending of GO film to the rGO side will make the sheets in rGO circuit compact together (Figure S18), leading to the electrical resistance decrease of about $-2.1 \%$ (blue line in Figure S17b). So the electrical resistance change of the built-in rGO sensor in Figure $3 \mathrm{e}$ in the main text is the result of a combination of the film deformation and surrounding humidity changes.
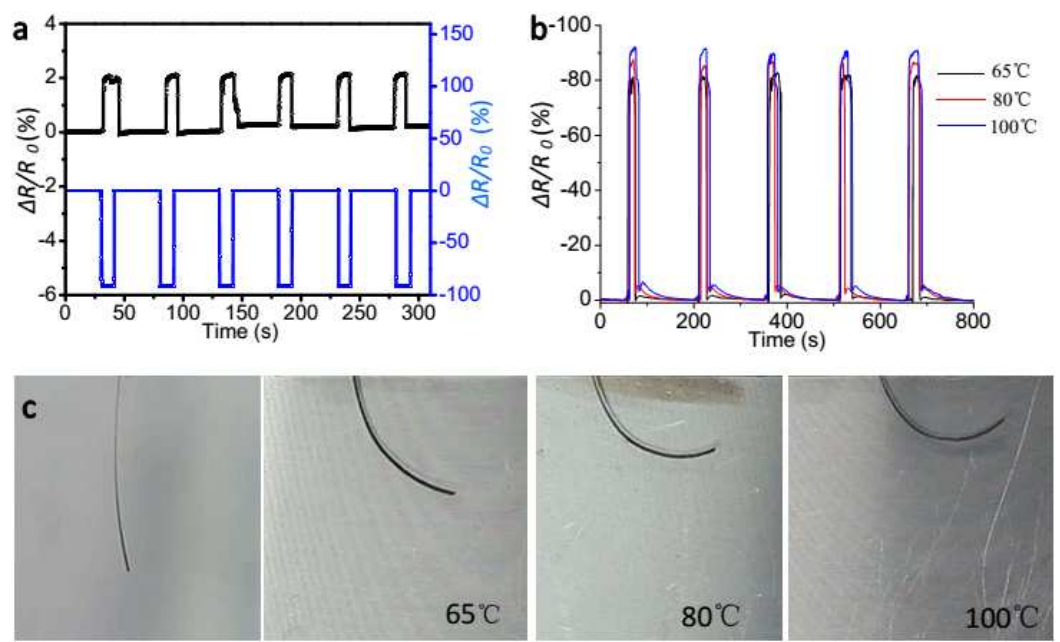

Figure S20. (a) The electrical resistance changes of the built-in rGO sensor when bending the film to the rough (black line) surface without temperature change. Temperature changes (room temperature to $100{ }^{\circ} \mathrm{C}$ ) induced the electrical resistance changes of the built-in rGO sensor in a fixed film (blue line). (b) The electrical resistance changes of the built-in rGO sensor when the free standing film was exposed to different temperatures. (c) The photos of the film's deformation when exposed to different temperatures. The marked temperature is the 
temperature of the heating panel behind the sample. The distance between the panel and sample is about $1 \mathrm{~mm}$.

Temperature increase can make $\mathrm{H}_{2} \mathrm{O}$ desorption in the film and decline the electrical resistance of the built-in rGO sensor (-95\%) in a fixed film (Figure S20a), which is much larger than the increased value $(2.1 \%)$ when the film bends to smooth surface without temperature changes (Figure S20a). As a result, the electrical resistance changes $\left(\Delta R / R_{0}\right)$ of the built-in sensor and the film bending curvature $(\theta)$ have a good synchronistical relationship (Figure S20b and c).

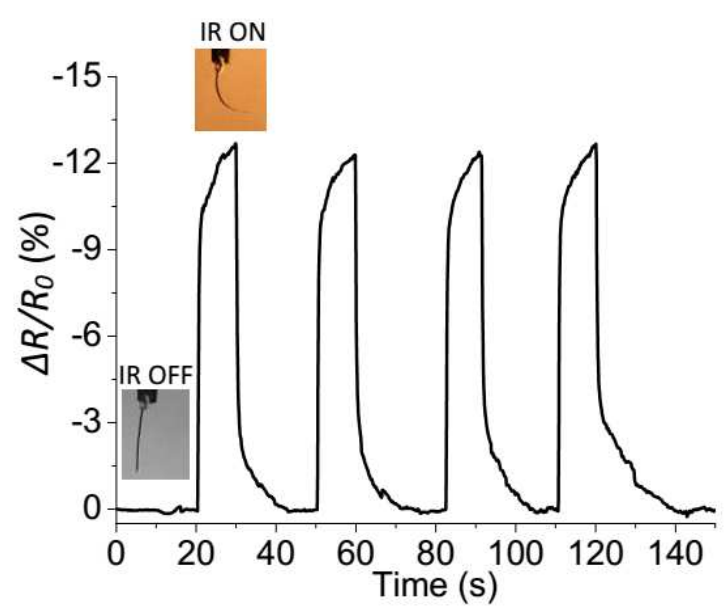

Figure S21. The electrical resistance changes of the built-in sensor when the film exposed to IR. Insert is the corresponding photos under different states.

When the film is exposed to the IR light, water desorption makes the film bend to the rough side and the electrical resistance of the built-in sensor decreases. (Figure S21) The electrical resistance changes of the built-in sensor increase means that the film is recovering to the initial state. 

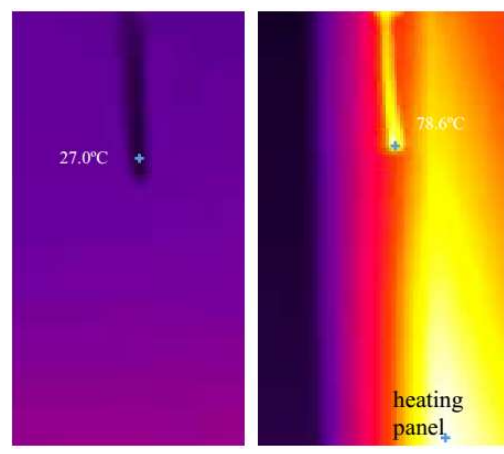

Figure S22. Thermal image of the film closed to a $100^{\circ} \mathrm{C}$ heating plane.
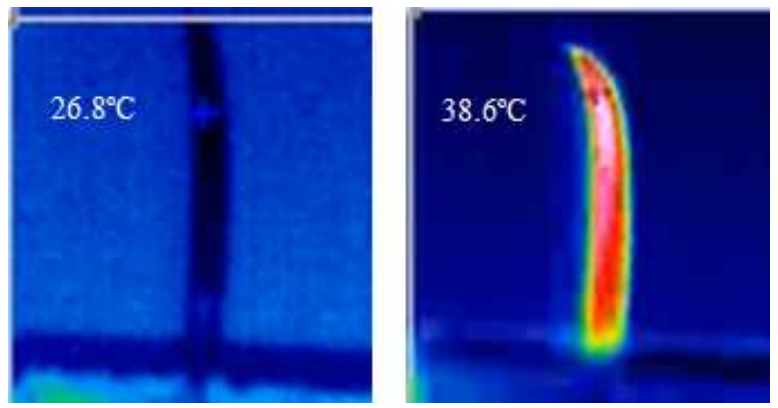

Figure S23. Thermal image of the film exposed to IR.

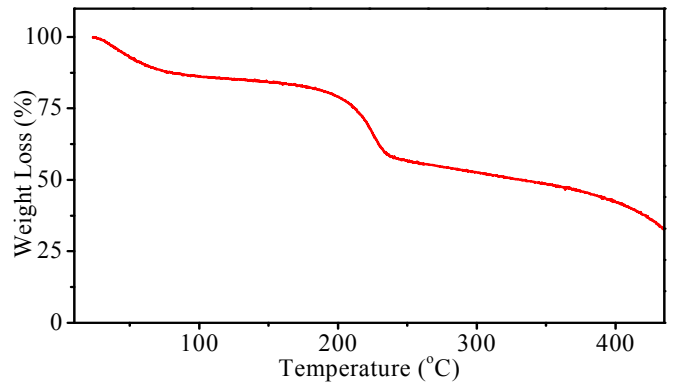

Figure S24. Thermal Gravimetric analysis (TGA) of GO.
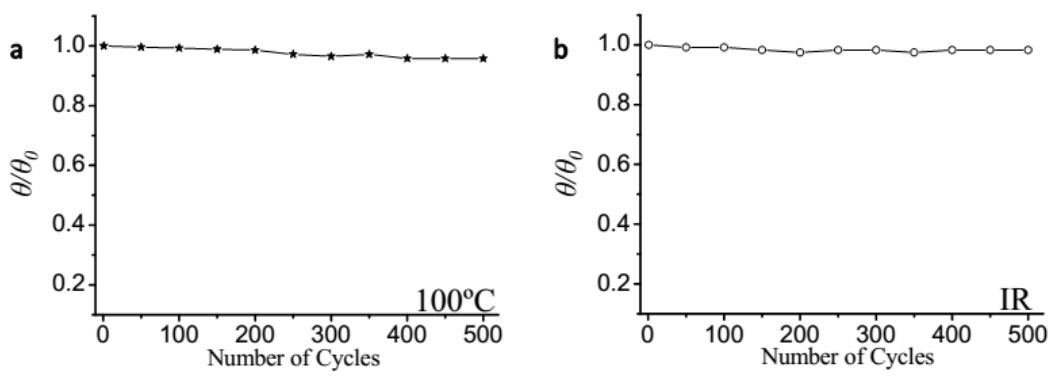

Figure S25. The cycling test of the actuator exposed to the heating plane (a) and IR (b). 


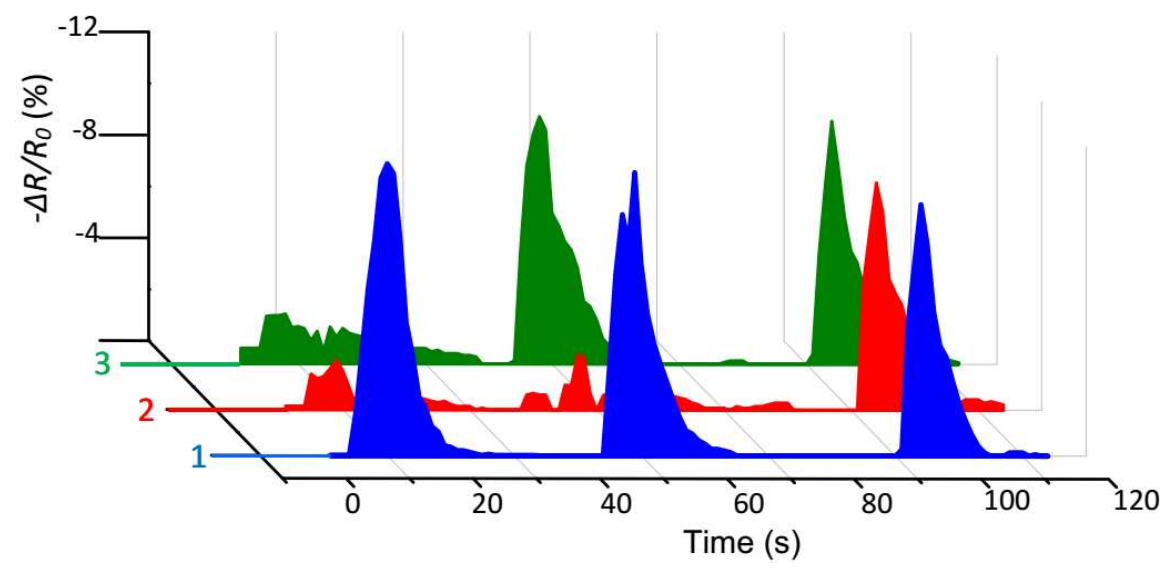

Figure S26. The electrical resistance changes of the built-in rGO sensor in three finger responsive device in Fig. 4c. Curve 1,2 and 3 corresponds to NO.1, 2 and 3 finger, respectively.

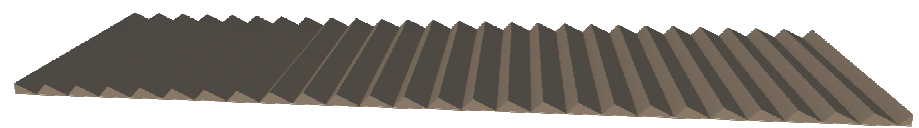

Figure S27. The schematic diagram of the ratchet substrate. Each ratchet in the substrate has two sides with different slops.

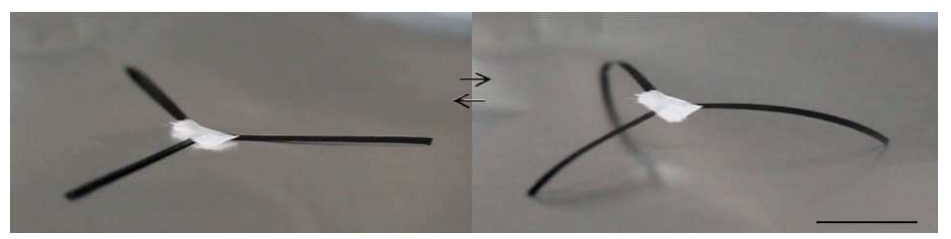

Figure S28. Three feet crane lifting and laying down a paperboard with the same weight as the crane when the temperature changes. Scale bars: $1 \mathrm{~cm}$. 


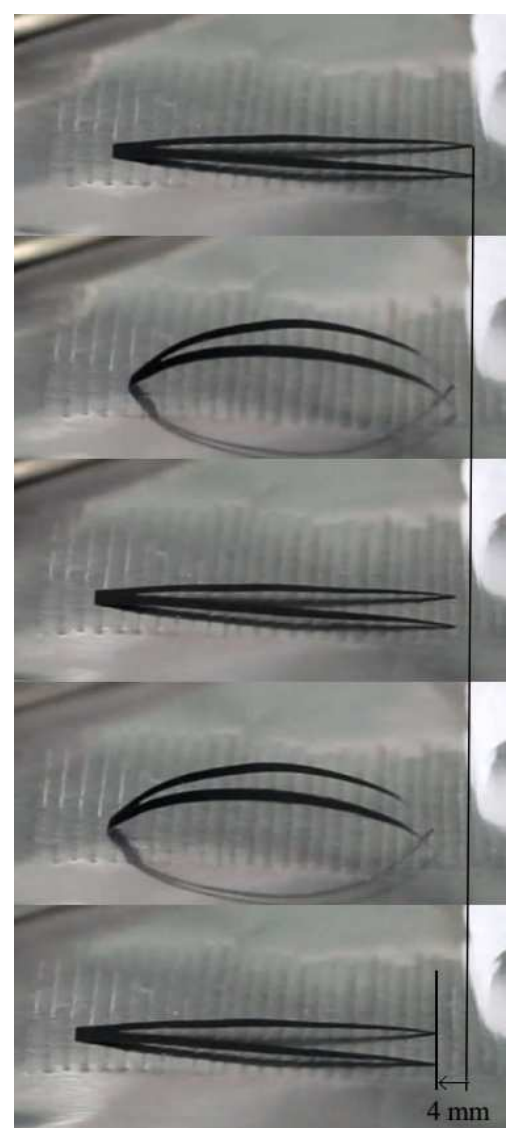

Figure S29. A series of photographs of a bipedal "reptile" moving forward when alternating the temperature (Room temperature to $100{ }^{\circ} \mathrm{C}$ ).

Once a heat panel $\left(100{ }^{\circ} \mathrm{C}\right)$ is approaching to the substrate, the reptile begins to bend and the back end of the legs shifts. Then the front side move towards when the reptile recovers to the initial flat state after the heat panel moving away, because the backside of body is fixed by the large slope side of the ratchet. With the alternation of the heat panel near and far away, the device walks forward step by step (Figure S29). After two steps, the reptile can move forwards about $4 \mathrm{~mm}$. 


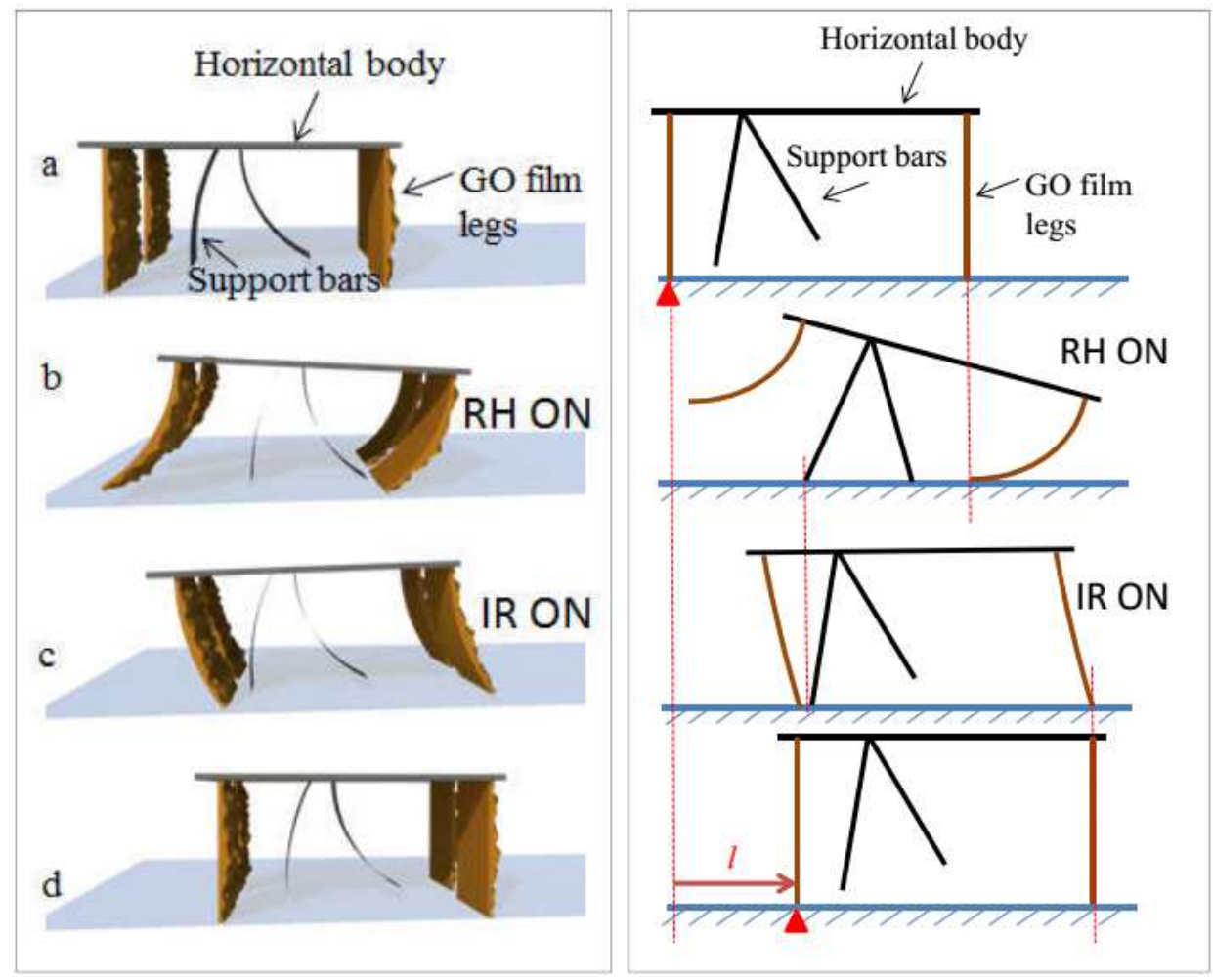

Figure S30. Schematic of the moving four feet walker. The left half of the diagram is the Three-Dimensional Drawings. The right half of the diagram is the corresponding Side-Effect Diagram. (a) The device is at static state. The device is supported by the GO film legs. (b) When the RH increases to about $80 \%$, the GO film legs bend and the device move forward until the support bars contact the ground and support the whole device. In this state, the GO film hind legs are lifted. (c) When the IR is on, all the GO film legs begin to recover to the initial state. In this process, the GO film hind legs contact the ground again. The whole device will move forwards after the legs recover to the initial state (d). $l$ is the distance that the device advances per cycle. 

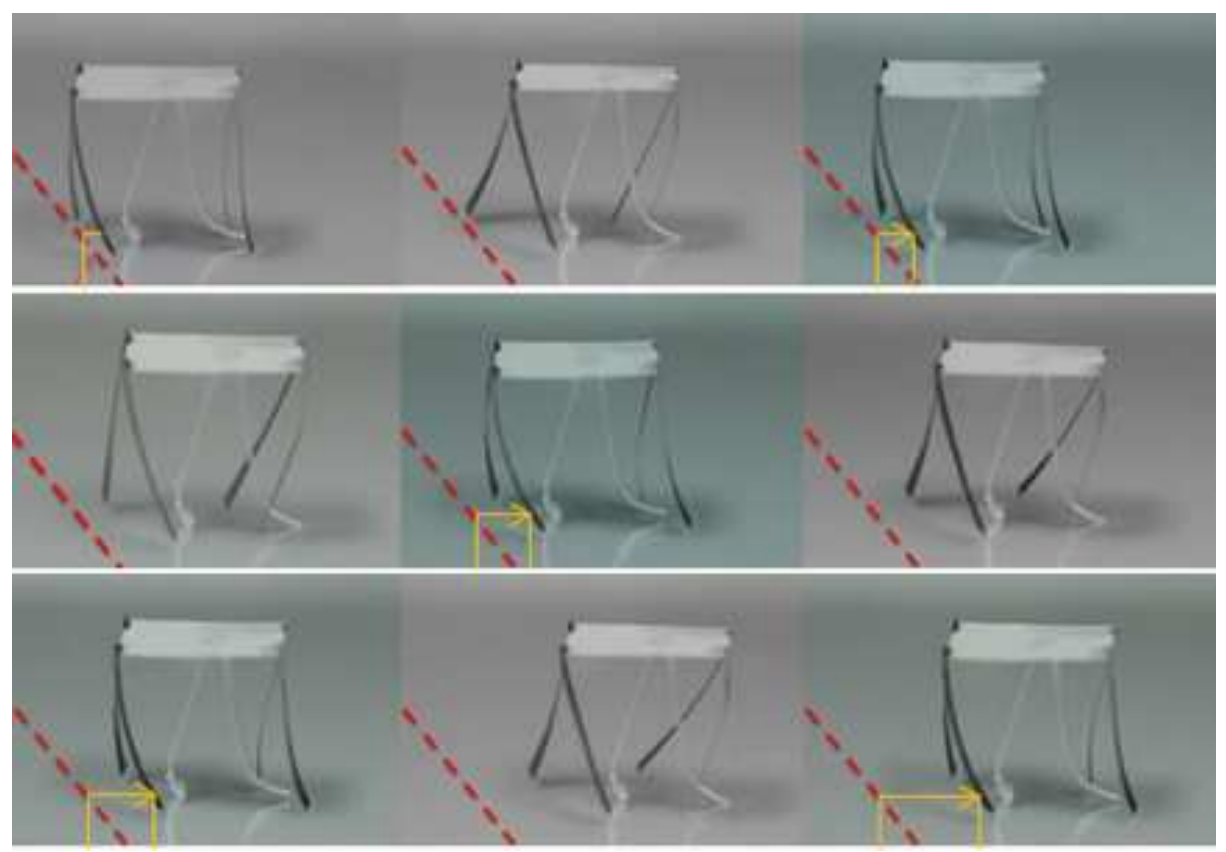

Figure S31. A series of photographs of a four-legged walker moving toward on a smooth glass.

Movie S1. A three finger responsive device.

Movie S2. A "rectangle" walker.

Movie S3. Four-legged walking robots.

\section{References}

s1. H. H. Cheng, Y. Hu, F. Zhao, Z. L. Dong, Y. H. Wang, N. Chen, Z. P. Zhang and L. T. Qu. Moisture-Activated Torsional Graphene-Fiber Motor. Adv. Mater. 2014, 26, 29092913.

s2. Y. Li, Y. Zhao, H. H. Cheng, Y. Hu, G. Q. Shi, L. M. Dai and L. T. Qu. Nitrogen-Doped Graphene Quantum Dots With Oxygen-Rich Functional Groups. J. Am. Chem. Soc. 2012, $134,15-18$. 
s3. Xie, X. J.; Qu, L. T.; Zhou, C.; Li, Y.; Zhu, J.; Bai, H.; Shi, G. Q.; Dai, L. M. An Asymmetrically Surface-Modified Graphene Film Electrochemical Actuator. ACS Nano 2010, 4, 6050-6054.

s4. Kim, K. S.; Zhao, Y.; Jang, H.; Lee, S. Y.; Kim, J. M.; Kim, K. S.; Ahn, J. H.; Kim, P.; Choi, J. Y.; Hong, B. H. Large-Scale Pattern Growth of Graphene Films Forstretchable Transparent Electrodes. Nature 2009, 457, 706-710

s5. Yan, C. Y.; Wang, J. X; Kang, W. B; Cui, M. Q.; Wang, X.; Foo, C. Y.; Chee, K. J.; Lee, P. S. Highly Stretchable Piezoresistive Graphene-Nanocellulosenanopaper for Strain Sensors. Adv. Mater. 2014, 26, 2022-2027.

s6. Borini, S.; White, R.; Wei, D.; Astley, M.; Haque, S.; Spigone, E.; Harris, N.; Kivioja, J.; Ryhanen, T. Ultrafast Graphene Oxide Humidity Sensors. ACS Nano 2013, 7, 1116611173.

s7. Medhekar, N. V.; Ramasubramaniam, A.; Ruoff, R. S.; Shenoy, V. B. Hydrogen Bond Networks in Graphene Oxide Composite Paper: Structure and Mechanical Properties. ACS Nano 2010, 4, 2300-2306. 